\title{
BEARING CAPACITY ANALYSIS OF FOUDATIONS ON SLOPES BY USE OF LOG-SPIRAL SLIDING SURFACES
}

\author{
Kunitomo Narita ${ }^{\mathrm{i})}$ and $\mathrm{H}_{\text {akuju }}$ Yamaguchi $^{\mathrm{i} i)}$
}

\begin{abstract}
This paper focuses on an extension of the log-spiral analysis of bearing capacity that has been presented in the authors' previous work for strip foundations placed on the level ground, to those on the top of slopes. Comparisons are made with other analytical and experimental results to examine applicability of the method to practical problems.

It is revealed that the log-spiral analysis somewhat overestimates bearing capacity values as compared to other solutions, the errors involved being around 20 percent at maximum and varying sensitively to the angle of internal friction and slope inclination. Also noticed is a relatively good correspondence with experimental results, especially with model tests on clay $(\phi=0)$, on both the ultimate bearing capacity and the shape of sliding surfaces.
\end{abstract}

Key words : bearing capacity, footing, shallow foundation, slip surface, slope stability, vertical load (IGC: $\mathrm{E} 3 / \mathrm{E} 6 / \mathrm{H} 1)$

\section{INTRODUCTION}

The authors have presented in their previous work (Narita and Yamaguchi, 1989) a conventional and practically useful analytical method of bearing capacity of foundations placed on the level ground, assuming that a sliding surface in the ground is composed of single log-spiral. Through comparisons with other theoretical and empirical formulae, it has been concluded that log-spiral bearing capacity factors are comparable to other theoretical values so far presented, and that the present analysis substantiates Meyerhof's hypothesis of the effective width in eccentric loading as well as validity of the theorem of correspondence and that of superposition in the calculation of the bearing capacity. This paper focuses on an extension of the presented log-spiral analysis to the bearing capacity of strip footings placed on the top of slopes.

Theoretical studies on the basis of plastic equilibrium by Meyerhof (1957), Sokolovski (1960) and Chen (1969) are representative on

i) Professor, Department of Civil Engineering, Aichi Institute of Technology, Toyota-shi, Aichi.

ii) Professor, Department of Construction Engineering, Faculty of Science and Engineering, Tokyo Denki University, Ishizaka, Hatoyama-cho, Hiki-gun, Saitama.

Manuscript was received for review on July 27, 1989.

Written discussions on this note should be submitted before April 1, 1991, to the Japanese Society of Soil Mechanics and Foundation Engineering, Sngayama Bldg. 4F, Kanda Awaji-cho 2-23, Chiyodaku, Tokyo 101, Japan. Upon request the closing date may be extended one month. 
this subject, though they are not much available for practical problems because of their limited analytical conditions. Kusakabe et al. (1981), on the other hand, presented a relatively simple and useful upper bound solution by constructing failure surfaces with two straight lines and a log-spiral. The usefulness of the solution was examined through comparisons with analytical and experimental results, and computed results were arranged in forms of charts for design purposes. Kusakabe (1985) later extended the procedure to slopes of small fills where base failure might be expected and to cut slopes of normally consolidated clay of strength increasing linearly with depth.

Experimental investigations have also been conducted on the problem by Shields et al. (1977), Akai et al. (1980) and Kusakabe et al. (1981) for ordinary 19-gravitational model fills, and Saitoh et al. (1981) and Terashi et al. (1986) for centrifuge models. Bearing capacity of a footing with an uneven base on slope has been one of recent related topics encountered in the field. Laboratory model tests and large scale field tests have been carried out to study practical evaluation of the bearing capacity of such footings (Keto et al., 1989).

In the present paper, numerical characteristics and applicability of the log-spiral solutions to practical problems are examined by comparing them with other analytical and experimental works listed above.

\section{LOG-SPIRAL ANALYSIS OF BEARING CAPACITY}

Toe and Slope Failures

In Fig. 1 a continuous sliding surface in the ground is assumed to be a log-spiral AE of its pole at point $O$ and starting from the left edge of a strip footing of width $B=2 b$, which rests on the top of a slope of inclination $\beta$ apart from the slope shoulder $D$ by a distance $L=\lambda B$. Putting $\mu=\tan \phi$ ( $\phi$ : angle of internal friction) and $r_{0}=\mathrm{OA}$, the equation of a $\log -$ spiral is written as $r=r_{0} \exp (\mu \theta)$, and a logspiral sliding surface can be defined by specifying sets of values about footing condition $(\beta$, 2) and the location of the pole $\mathrm{O}\left(r_{0}, \alpha\right)$.

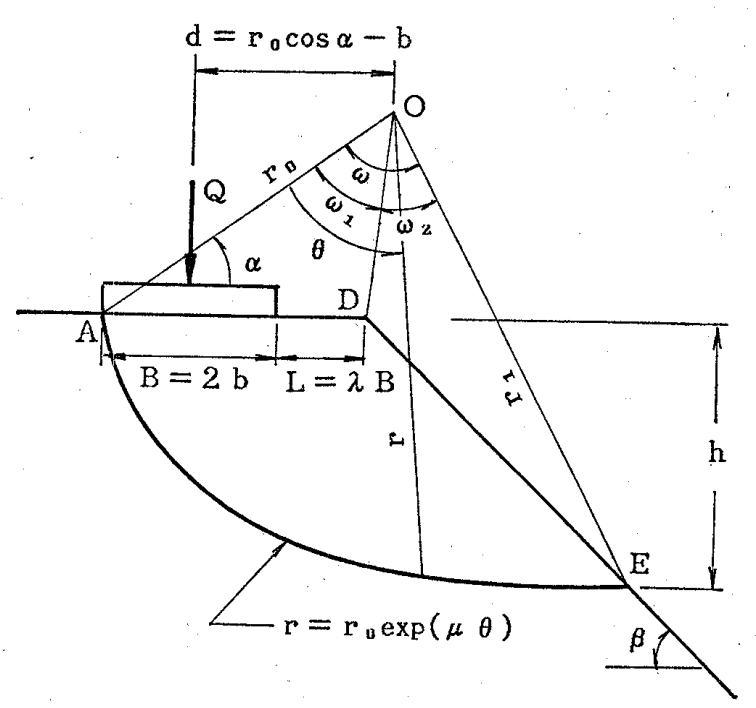

Fig. 1. Log-spiral sliding surface

Geometrical conditions in triangles OAD and ODE yield an equation

$$
\begin{aligned}
\frac{B+L}{\sin \omega_{1}} & =\frac{r_{0}}{\sin \left(\alpha+\omega_{1}\right)} \\
& =\frac{r_{0} \exp (\mu \omega) \sin (\alpha+\beta+\omega)}{\sin \alpha \sin \left(\alpha+\beta+\omega_{1}\right)}
\end{aligned}
$$

The central angle $\omega$ that locates the end point $\mathrm{E}$ of the spiral is then given by eliminating $\omega_{1}$ as a function of the above four variables in the form

$$
\begin{aligned}
& \exp (\mu \omega) \sin (\alpha+\beta+\omega) \\
& \quad=\sin (\alpha+\beta)-B(1+\lambda) \sin \beta / r_{0}
\end{aligned}
$$

For the level ground $(\beta=0)$, Eq. (1) reduces to a simple expression of the form (Narita and Yamaguchi, 1989)

$$
\exp (\mu \omega)=\sin \alpha / \sin (\alpha+\omega)
$$

where $\omega$ is determined from $\alpha$ irrespective of $\lambda$ and $r_{0}$.

The ultimate bearing capacity of a strip footing is generally represented as a linear combination of the cohesion $c$, the surcharge load $p_{0}$ and the weight of soil $\gamma$. In the present logspiral analysis, each component of the ultimate load $Q\left(Q_{c}, Q_{q}\right.$ and $\left.Q_{r}\right)$ is determined by minimizing corresponding footing load that is derived individually from the equilibrium of moments about pole $O$. The ultimate load of $p_{0}$-term, $Q_{q}$, is not considered in this paper for convenience in practical application. The effect of foundation depth on the bearing capac- 
ity of deep foundations will later be discussed briefly.

For an infinitely long slope shown in Fig. 1, where toe or slope failure is anticipated, the derivation of $Q_{c}$ and $Q_{\gamma}$ is presented as follows.

(1) $Q_{c}$ : The equilibrium of moments about pole $O$ of the cohesion $c$ is written in the same form as presented in the case of the level ground, that is

$$
Q_{c} \times d=\int_{0}^{\omega} c r^{2} d \theta=c r_{0}^{2} \chi / 2 \mu
$$

where $d=r_{0} \cos \alpha-b, \chi=\exp (2 \mu \omega)-1, \quad$ and the value of $\omega$ is determined from Eq. (1). For purely cohesive $\phi=0$ materials, a logspiral degenerates into a circle and $\mu=\chi=0$. The resisting moment in this case is given by $c r_{0}^{2} \omega$ which results in $\chi / 2 \mu \rightarrow \omega$ in Eq. (3).

(2) $Q_{r}$ : Denoting the moment of the weight relative to pole $O$ of the semicircular portion between $\mathrm{OA}$ and $\mathrm{OE}$ by $M_{r 1}$ and that of polygon OADE by $M_{72}$, clockwise moment being positive, the equilibrium gives an equation

$$
\begin{aligned}
Q_{r} \times d= & M_{r^{1}}-M_{\gamma 2} \\
= & -\left(\gamma r_{0}^{3} / 6\right)\{g(\alpha)-h(\alpha)\} \quad(4) \\
g(\alpha)= & \left(2 / 1+9 \mu^{2}\right)[\exp (3 \mu \omega)\{3 \mu \cos (\alpha+\omega) \\
& +\sin (\alpha+\omega)-3 \mu \cos \alpha-\sin \alpha] \\
h(\alpha)= & {\left[\exp (2 \mu \omega) \sin \omega_{2} \cos (\alpha+\omega)\right.} \\
& +\exp (\mu \omega) \sin \omega_{2} \sin \alpha \\
& \left.+\sin \omega_{1} \sin \left(2 \alpha+\omega_{1}\right) / \sin \left(\alpha+\omega_{1}\right)\right] \\
& \times \sin \alpha / \sin \left(\alpha+\omega_{1}\right)
\end{aligned}
$$

According to the previous work (Narita and Yamaguchi, 1989), the ultimate load is to be obtained by applying two minimizing conditions with respect to $\left(r_{0}, \alpha\right)$, though the derivation is in reality very to complicated to have a final closed analytical solution. Numerical computations are therefore unavoidable to find minimum value of $Q\left(=Q_{c}+Q_{r}\right)$ in a similar way as adopted in routine calculation of stability analysis of slopes. The ultimate bearing capacity $q=Q / B$ thus obtained without the surcharge term can be represented by use of bearing capacity factors $N_{c}$ and $N_{r}$ in the form

$$
q / \gamma B=(c / \gamma B) N_{c}+N_{r} / 2
$$

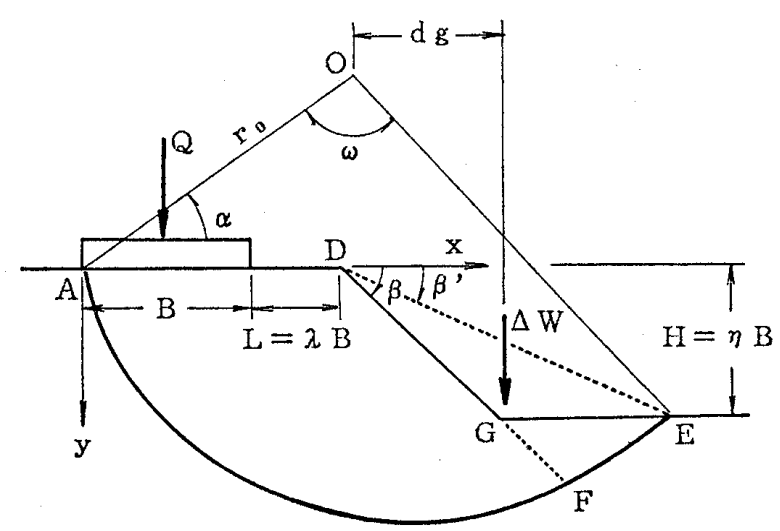

Fig. 2. Analysis of base failure

\section{Base Failures}

For a relatively small fill of the height $H=$ $\eta B$ where the base failure might be predominant, the above derivation requires a little modification, as shown in Fig. 2. It should be judged first of all whether the base failure takes place or not for given slope and footing conditions. This is done by determining the location of the point of intersection $F$ of the log-spiral and the extension of slope surface DG of inclination $\beta$; i. e., the base failure appears when point $F$ is located below the toe $\mathrm{G}\left(y_{F}>y_{G}=H\right)$. The value of the central angle $\omega$ in this case can be obtained numerically under conditions that $y_{\mathrm{E}}=H$ at point $\mathrm{E}$, that is

$$
\exp (\mu \omega) \sin (\alpha+\omega)-\sin \alpha=H / r_{0}
$$

The expression of $Q_{c}$ does not change from Eq. (3) for the base failure. The footing load $Q_{r}$ calculated by Eq. (4) with the $\omega$-value determined from Eq. (6) is equivalent to that for an infinite slope of inclination $\beta^{\prime}$ which passes through the point E. $Q_{\gamma}$-value thus obtained should therefore be corrected by an amount corresponding to the weight of soil $\Delta W$ of the triangle DGE. The additional footing load $\Delta Q_{\gamma}$ to be adjusted is then given by

$$
\Delta Q_{r} \times d=-\Delta W \times d_{g}
$$

\section{NUMERICAL RESULTS AND DISCUS- SIONS}

Kusakabe et al. (1981) have presented a simple upper bound solution for the bearing capacity of foundations on slopes, by introduc- 


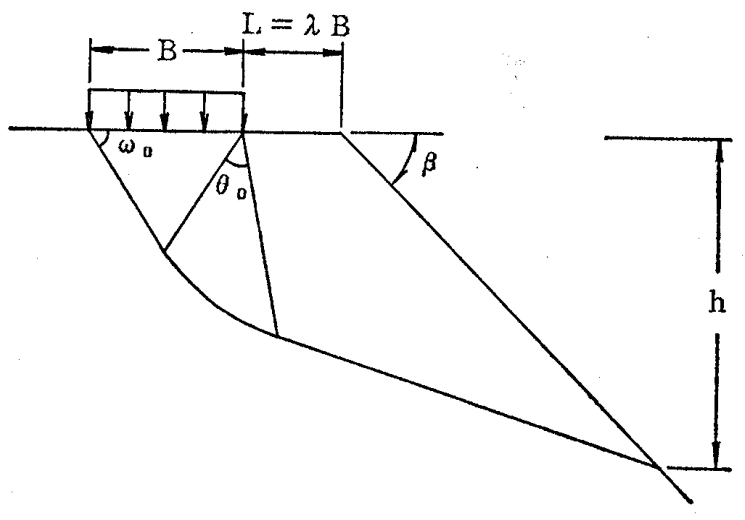

Fig. 3. Failure mechanism for upper bound analysis (Kusakabe et al.)

ing a failure mechanism which consists of a triangular active wedge, a log-spiral radial shear zone and a quadrilateral passive zone, as shown in Fig. 3. The usefulness of the solution has been proved by comparing with other analytical solutions and experimental results. Numerical results of the present log-spiral analysis are studied in the followings, together with the above upper bound solutions, to discuss its characteristics and applicability to practical problems.
Comparisons with Other Analytical Solutions

Numerical results of normalized bearing capacity $q / \gamma B$ from the log-spiral and the upper bound analyses and those by the simplified Bishop method of slices are compared in Table.1 for various conditions of foundation soil $(c$, $\phi$ ) and footing $(\beta, \lambda)$ : i. e., variations of $q$ / $\gamma B$ due to $(c / \gamma B, \phi)$ are examined in rows I and II for a condition $\left(\beta=30^{\circ}, \lambda=0\right)$ and those due to $(\beta, \lambda)$ in rows III and IV for $(c /$ $\left.\gamma B=1, \phi=30^{\circ}\right)$. It is noticed that in most of cases the log-spiral analysis gives the largest value of $q / \gamma B$ of the three solutions, followed by the simplified Bishop method. The values indicated in parentheses on the column of logspiral reveal that its error from the upper bound solution is considerably affected by the angle of internal friction $\phi$ and the slope inclination $\beta$ rather than $c / \gamma B$ and $\lambda$.

For purely cohesive $\phi=0$ materials where a log-spiral degenerates into a circle, the logspiral solutions become almost equal to the values by the Bishop method and at most 3 5 percent larger than the upper bound solutions. Incidentally, the ratio of $N_{c}$-values of the log-

Table 1. Comparisons of analytical solutions

\begin{tabular}{|c|c|c|c|c|c|c|c|}
\hline & \multirow{2}{*}{$c / r B$} & \multirow{2}{*}{$\phi$} & \multirow{2}{*}{$\beta$} & \multirow{2}{*}{$\lambda$} & \multicolumn{3}{|c|}{$q / \gamma B$} \\
\hline & & & & & Log-Spiral & Bishop & Upper Bound \\
\hline \multirow{4}{*}{ I } & 25 & \multirow{4}{*}{0} & \multirow{4}{*}{$30^{\circ}$} & \multirow{4}{*}{0} & $107.0(1.05)$ & $104.4(1.02)$ & 102.0 \\
\hline & 5 & & & & $21.1(1.04)$ & $20.8(1.03)$ & 20.2 \\
\hline & 1 & & & & 3. $94(1.03)$ & $4.03(1.05)$ & 3.84 \\
\hline & 0.5 & & & & - & $1.92(1.08)$ & 1.78 \\
\hline \multirow{4}{*}{ I } & 25 & \multirow{4}{*}{$30^{\circ}$} & \multirow{4}{*}{$30^{\circ}$} & \multirow{4}{*}{0} & 449.9 (1.14) & $424.7(1.08)$ & 395.0 \\
\hline & 5 & & & & $92.3(1.14)$ & $86.3(1.07)$ & 81.0 \\
\hline & 1 & & & & $20.7(1.13)$ & $18.6(1.02)$ & 18.3 \\
\hline & 0.5 & & & & $11.7(1.14)$ & $10.2(0.99)$ & 10.3 \\
\hline \multirow{4}{*}{ III } & \multirow{4}{*}{1} & \multirow{4}{*}{$30^{\circ}$} & $15^{\circ}$ & \multirow{4}{*}{0} & $35.2(1.22)$ & $29.0(1.00)$ & 28.9 \\
\hline & & & $30^{\circ}$ & & 20.7 (1.13) & $18.6(1.02)$ & 18.3 \\
\hline & & & $45^{\circ}$ & & $12.1(1.06)$ & $12.0(1.05)$ & 11.4 \\
\hline & & & $60^{\circ}$ & & $7.11(1.01)$ & $8.10(1.15)$ & 7.06 \\
\hline \multirow{4}{*}{ N } & \multirow{4}{*}{1} & \multirow{4}{*}{$30^{\circ}$} & \multirow{4}{*}{$30^{\circ}$} & 0.5 & $24.5(1.15)$ & $21.4(1.00)$ & 21.3 \\
\hline & & & & 1 & $28.6(1.17)$ & $24.8(1.02)$ & 24.4 \\
\hline & & & & 2 & 35.5 (1.18) & $31.7(1.06)$ & 30.0 \\
\hline & & & & 4 & $49.6(1.19)$ & 48.1 (1.15) & 41.7 \\
\hline
\end{tabular}

( ): ratio to Upper Bound solution 
spiral to the upper bound analyses yields $5.52 /$ $5.14=1.07$ for the level ground $(\beta=0)$, which is the largest difference between the two. For the case where $\phi=30^{\circ}$, on the other hand, the log-spiral solutions differ much more from the upper bound ones; the difference becomes about 15 percent or more, irrespective of $c / \gamma B$, and increases slightly due to the increase in $\lambda$-value. Also noticed is the fact that the increase of $\beta$-value produces a large reduction of the difference, being accompanied by a serious decrease in the value of the ultimate bearing capacity.

\section{Comparisons with Test Results for Clay}

The log-spiral solutions are compared with the results of model tests and corresponding upper bound calculations presented by Kusakabe et al. (1981), as listed on Table. 2. In their work, model slopes of Kanto loam were loaded by jack on the top surfaces for different combinations of $(\beta, \lambda)$, and the results were compared with the theoretical estimates by the $\phi_{u}=0$ analysis because the undrained failure was considered more probable due to relatively high speed of loading. The log-spiral solutions given in Table. 2 were also evaluated by the $\phi_{u}=0$ analysis for circular sliding surfaces, and they show fairly good correspondence with the upper bound solutions as noted before.

Concerning the reasons why the test results are in excess of these theoretical estimates by around 30 percent, Kusakabe et al. (1981) have noticed that the effects of stress conditions on

Table 2. Comparisons with test results for clay

\begin{tabular}{|c|c|c|c|c|}
\hline \multirow{2}{*}{$\beta$} & \multirow{2}{*}{$\lambda$} & \multicolumn{3}{|c|}{ Bearing Capacity: $\mathrm{kN} / \mathrm{m}^{2}$} \\
\hline & & Test & Upper Bound & Log-Spira 1 \\
\hline \multirow{3}{*}{$30^{\circ}$} & 0 & 28.5 & 21.5 & $22.5(1.05)$ \\
\hline & 0.5 & 44.4 & 40.7 & $42.9(1.05)$ \\
\hline & 1.0 & 34.4 & 35.3 & $37.2(1.05)$ \\
\hline \multirow{3}{*}{$45^{\circ}$} & 0 & 32.2 & 20.7 & $21.4(1.03)$ \\
\hline & 0.5 & 30.8 & 27.2 & $28.2(1.04)$ \\
\hline & 1.0 & 38.3 & 26.5 & $27.4(1.03)$ \\
\hline \multirow{3}{*}{$60^{\circ}$} & 0 & 34.6 & 27.5 & $28.0(1.02)$ \\
\hline & 0.5 & 44.8 & 33.4 & $34.0(1.02)$ \\
\hline & 1.0 & 30.3 & 25.3 & $25.5(1.01)$ \\
\hline
\end{tabular}

(a) $\beta=60^{\circ}, \lambda=0.5$

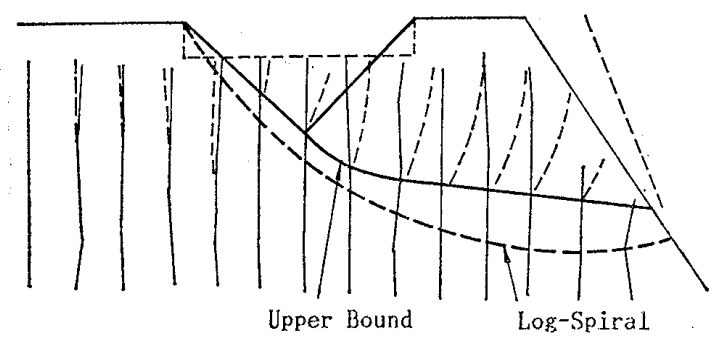

(b) $\beta=30^{\circ}, \lambda=0.5$

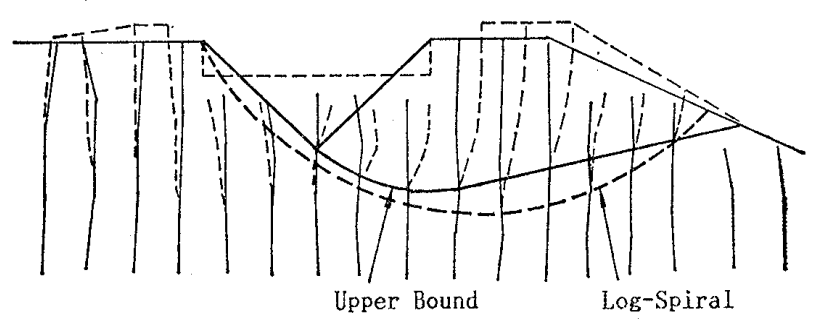

Fig. 4. Comparisons of sliding surfaces $(\phi=0)$

the undrained strength and the influence of side friction between the sample and the wall of the container are the most responsible.

Shapes of sliding surfaces are compared in Fig. 4 for cases of $\beta=30^{\circ}$ and $60^{\circ}$, by putting the log-spiral and the upper bound solutions by thick broken and solid lines, respectively, upon the observed deformation patterns. It is seen that both analytical sliding surfaces lie closely along from the active wedge to the radial shear zone, beyond which rather large gap is inevitable between the two.

\section{Comparisons with Test Results for Sand}

Saitoh et al. (1981) have performed a series of loading tests on model slopes of Toyoura sand $\left(\beta=25^{\circ} \sim 35^{\circ}\right)$ by use of a centrifuge and have demonstrated that the test results are in good accordance with those presented by Shields et al. (1977) on dense sand slopes of $\beta=26.6^{\circ}$. These test results are plotted on Fig. 5 together with the log-spiral and the upper bound solutions to be compared.

Numerical data herein employed for computations were determined to approximate the above test conditions as $\beta=25^{\circ}, c / \gamma B=0, \phi=40^{\circ}$ and $45^{\circ}$, and $\eta=H / B=2$ by which base failures are involved in the analysis. Concerning the value of $\phi$ of Toyoura sand, Saitoh et al. (1981) 


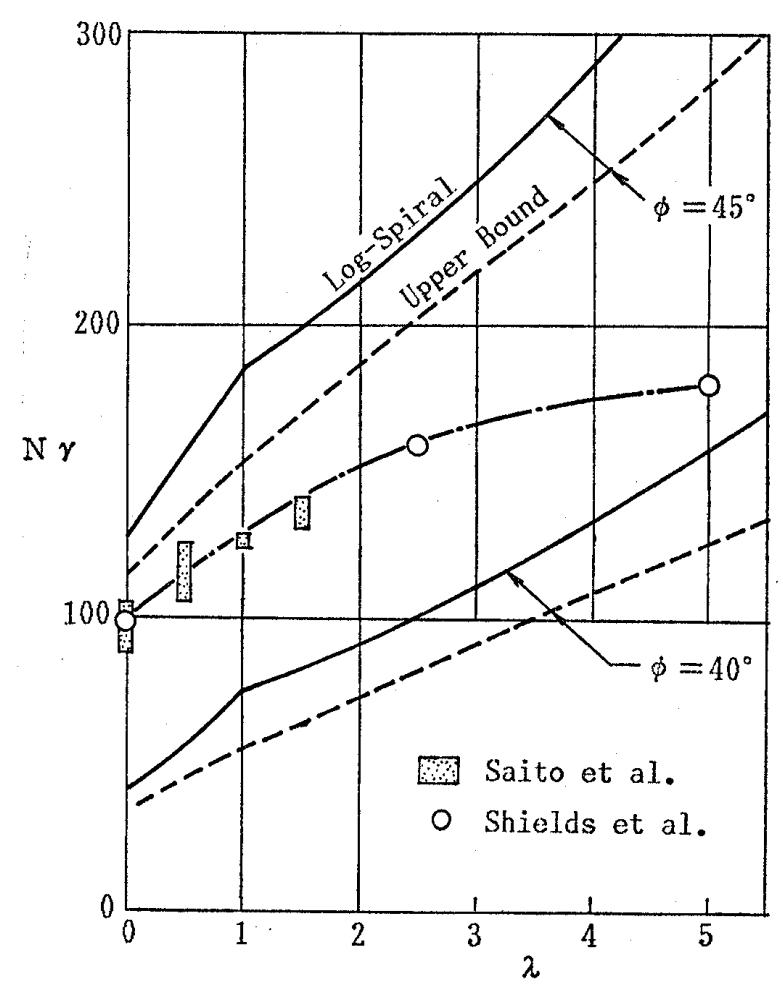

Fig. 5. Comparisons with test results for sand

obtained $\phi_{\max }=50^{\circ}$ through plane strain tests on specimens having the same density as used in model slopes, though a little reduction of $\phi$ should be considered (Yamashita et al., 1982) in order to introduce the influence of progressive failure in the analysis. The above-mentioned $\phi$-values, $40^{\circ}$ and $45^{\circ}$, correspond to 20 and 10 precent reduction of $\phi_{\max }=50^{\circ}$.

It can be recognized in Fig. 5 that both analytical solutions, different from the tendency of the test results, show successive increase in $N_{r}$-value as $\lambda$ increases, and that for small values of $\lambda$ relatively good qualitative and quantitative correspondence is attained between them. It is also noticed that the log-spiral relation curves of $N_{\gamma}$ versus $\lambda$ have a clear turning point at $\lambda \risingdotseq 1$ because patterns of sliding

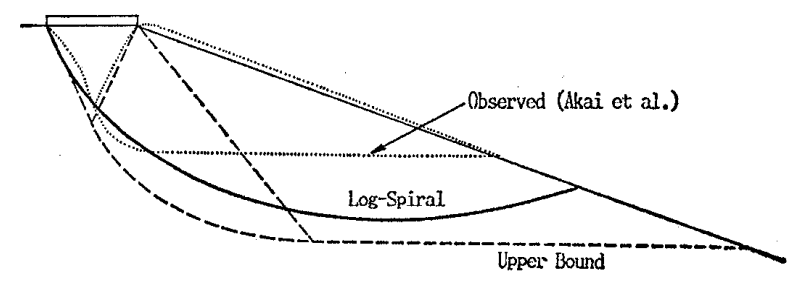

Fig. 6. Comparisons of sliding surfaces $(c=0)$ surface shift in turn as slope, toe and base failures near this point.

Shapes of sliding surfaces are compared in Fig. 6 for a result of model loading tests on sand slopes which Akai et al. (1980) have presented. The tests were conducted on slopes of dense Toyoura sand $(e \doteqdot 0.65)$ with $B=9.6$ $\mathrm{cm}, \lambda=0$ and $\beta$ changed in the range from $0^{\circ}$ to $30^{\circ}$. The observed sliding surface drawn by dotted lines in Fig. 6 is the one for $\beta=20^{\circ}$, which according to their comparison with the Sokolovski theoretical solutions resembles quite well to the solution for $\phi=40^{\circ}$. The log-spiral and the upper bound sliding surfaces are thus obtained for $\phi=40^{\circ}$ to be compared in Fig. 6 .

It is noticeable in the figure that both of analytical sliding surfaces substantially resemble in shape to the observed one but differ considerably from actual shallow sliding failure. This must be due to the fact that low confining pressure in the surface part of slope may cause local shear failure of sand, which really came out as an overall shallow surface sliding in the experiment.

\section{Analysis of Base Failures}

Yamashita et al. (1981, 1982) presented some analyses of bearing capacity of foundations on slopes by use of the rigid-body-spring model (RBSM) and compared with the upper bound solutions and the experimental results mentioned above. Fairly good agreement was then found for slope failures between the RBSM and the upper bound solutions with the difference of around 3 percent at most. Further development of the RBSM analysis was done for small fills where the base failure might be predominant. The results are plotted by open circles on Fig. 7 as a relationship between normalized value of the bearing capacity $q / \gamma B$ and the slope height $\eta=H / B$. Also drawn by solid and broken lines are the log-spiral and the upper bound solutions, respectively, for the same conditions as adopted in the RBSM analysis $\left(c / \gamma B=1, \phi=30^{\circ}, \beta=45^{\circ}\right.$ and $\left.\lambda=0\right)$.

It is seen in Fig. 7 that the values of $q / \gamma B$ of three different solutions wholly decrease with the increase in $\eta$ without any good order in magnitude: e. g., the log-spiral solution tends 


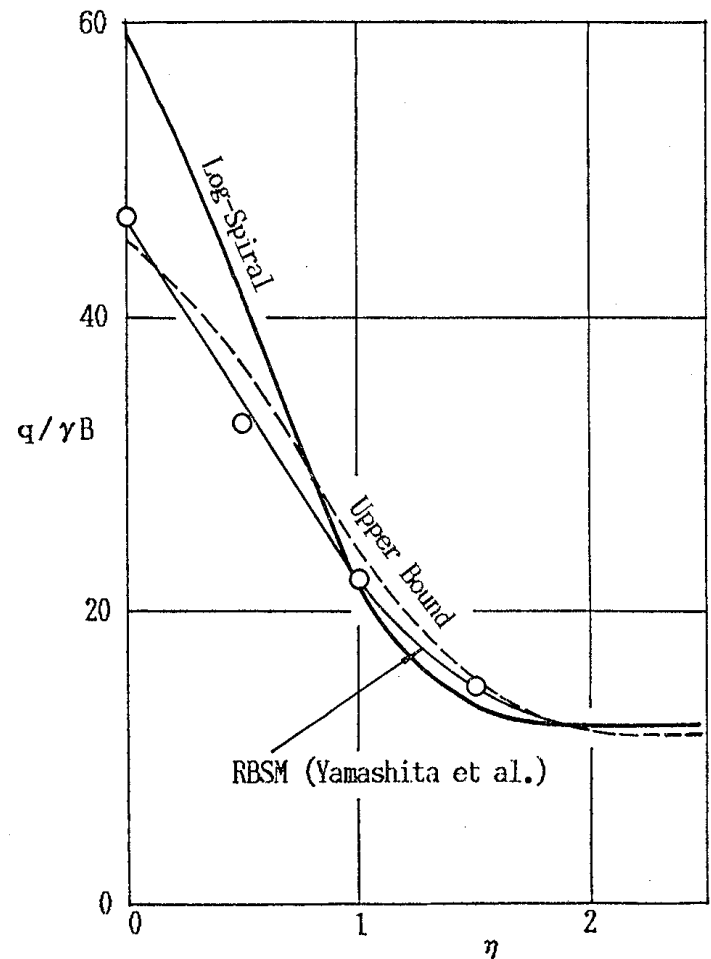

Fig. 7. Solutions for base failure

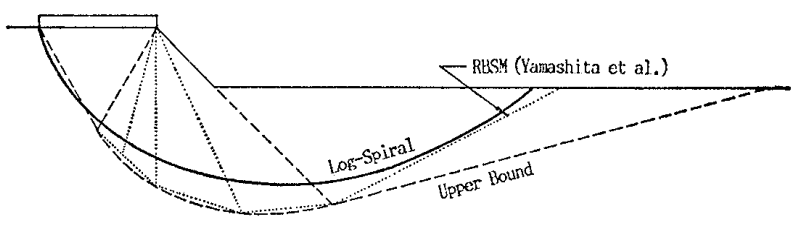

Fig. 8. Sliding surfaces of base failure

to have excessively high value of $q$ in the range $\eta<0.5$, but it gives value of $q$ lower than the other solutions in the range $\eta>1.0$.

Shapes of sliding surfaces of the above three solutions for $\eta=0.5$ are compared in Fig. 8 . It is recognized that the upper bound sliding surface coincides with the RBSM one in the active and radial shear zone but differs much from the others in the passive zone, and that the RBSM solution gives an intermediate sliding surface among them.

\section{The Effect of Foundation Depth}

The effect of foundation depth $D_{f}$ on the bearing capacity of deep foundations can readily be examined by introducing moment equilibrium for the weight of soil $\Delta W_{1}$ of the trapezoidal section efgh, as shown in Fig. 9. The

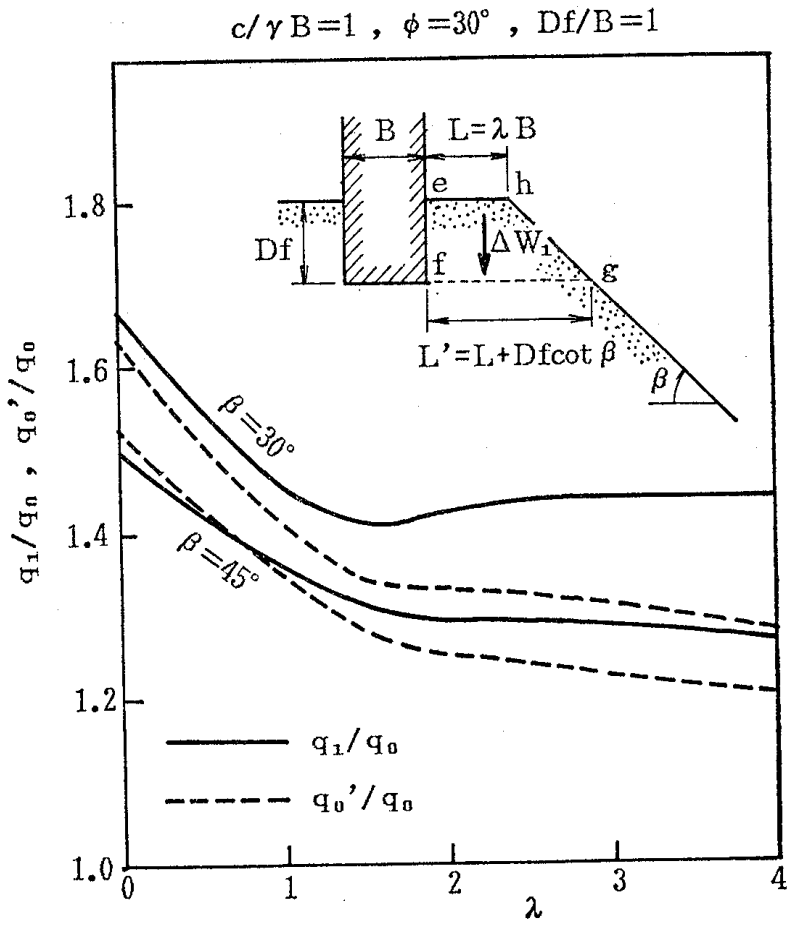

Fig. 9. Effects of foundation depth

increase of bearing load $\Delta Q_{q}$ to be in equilibrium with $\Delta W_{1}$ is given in the form: $\Delta Q_{q}=-\Delta W_{1}$ $\times d_{1} / d$, where $d_{1}$ and $d$ are the arm lengths of $\Delta W_{1}$ and $\Delta Q_{q}$, respectively, from pole $\mathrm{O}$. Corresponding normalized bearing capacity that should be added to Eq. (5) is then presented as

$$
\begin{aligned}
\Delta q / \gamma & B=-\left(D_{f} / B\right) \\
\times & \left\{\lambda+\left(D_{f} / B\right) \cot \beta / 2\right\} d_{1} / d
\end{aligned}
$$

Example calculation is done in Fig. 9 for the depth ratio $D_{f} / B=1$, in cases of $c / \gamma B=$ $1, \phi=30^{\circ}$ and $\beta=30^{\circ}, 45^{\circ}$. Solid lines represent variations of the ratio of bearing capacity $q_{1}$ of $D_{f} / B=1$ to $q_{0}$ of $\mathrm{D}_{f} / B=0$, that is, variations of the rate of increase in the bearing capacity due to foundation depth with the value of $\lambda$. It is recognized that larger effect of foundation depth is expected for smaller value of $\lambda$ in the range $\lambda<1.5$.

It might be reasonable to believe that the above-mentioned increase in the bearing capacity is based on combined effects of overburden load on the foundation level and an apparent enlargement of the distance of the base from the slope shoulder from $L$ to $L^{\prime}=L+D_{f} \cot \beta$. The latter effect is examined numerically by 
comparing bearing capacity values at $D_{f} / B=0$ as an increasing rate of bearing capacity $\left(q_{0}{ }^{\prime}\right)$ $q_{0}$ ) due to the increase in the base distance from $L$ to $L^{\prime}$, as shown by broken lines in Fig. 9. It should be noted that bearing capacity ratios thus represented by solid and broken lines vary in a similar manner with $\lambda$ for small values of $\lambda$, which leads to a conclusion that most of the effect of foundation depth is based on an apparent enlargement of the distance of the base from the slope shoulder.

Numerical investigations further confirmed that the ordinary way of introducing the effect of foundation depth into the analysis as the overburden load $p_{0}=\gamma D_{f}$ on the foundation level $f_{g}$ produces almost the same results as obtained in the present analysis where the exact shape of the weight $\Delta W_{1}$ is considered, the difference between them being around 5 percent at most.

\section{CONCLUSIONS}

Concluding remarks drawn from the present study are summarized in the following:

(1) The log-spiral analysis tends to overestimate bearing capacity values as compared to the upper bound and the simplified Bishop solutions. The errors involved are around 20 percent at maximum and vary sensitively to the angle of internal friction $\phi$ and slope inclination $\beta$.

(2) For purely cohesive $\phi=0$ materials where a log-spiral degenerates into a circle, the log-spiral solutions become close to other analytical solutions with the errors of 5 percent at most. They also show a relatively good correspondence with the results of model tests on clay with respect to the shape of sliding surface as well as the ultimate load.

(3) For cohesionless soils, both the logspiral and the upper bound solutions show successive increase in $N_{r}$-value as $\lambda$ increases, which yields much difference from the test results for large value of $\lambda$.

(4) The log-spiral solutions also show a fairly good correspodence with other analytical results in cases where base failures are anticipated to occur, giving rather lower value of the ultimate load than the upper bound solutions in a certain range of the slope height.

(5) Most of the effect of foundation depth on the bearing capacity of deep foundations is based on an apparent enlargement of the distance of the base from the slope shoulder, which leads to an increase in the bearing capacity.

\section{ACKNOWLEDGEMENTS}

The authors would like to express their sincere thanks to Dr. O. Kusakabe, Associate Professor of Utsunomiya University, for his valuable help to this investigation.

\section{REFERENCES}

1) Akai, K., Sano, I. and Imahori, Y. (1980): "The bearing capacity of foundation on the inclined surface (Model tests)," 15th Annual Meeting of JSSMFE, pp. 901-904 (in Japanese).

2) Chen, W. F. (1969): "Soil mechanics and theorems of limit analysis," Proc. of ASCE, Vol. 95, SM1, pp. 493-518.

3) Keto, H., Maeda, Y., Uehara, S. and Kusakabe, O. (1989): "A practical evaluating method for bearing capacity of spread foundation on slope," Proc. of JSCE, No. 403, VI-10, pp. 147-156 (in Japanese).

4) Kusakabe, O., Kimura, T. and Yamaguchi, H. (1981): "Bearing capacity of slopes under strip loads on the top surfaces," Soils and Foundations, Vol. 21, No. 4, pp. 29-40.

5) Kusakabe, O.(1985): "Some calculation on bearing capacity of strip footings on slopes," Tsuchito-Kiso, JSSMFE, Vol. 23, No. 2, pp. 7-12 (in Japanese).

6) Meyerhof, G. G. (1957): "The ultimate bearing capacity of foundations on slopes," Proc. 4th ICSMFE, Vol. 1, pp. 384-386.

7) Narita, K. and Yamaguchi, H. (1989): "Analysis of bearing capacity for log-spiral sliding surfaces," Soils and Foundations, Vol. 29, No. 2, pp. 85-98.

8) Saitoh, K., Shinjo, K., Yamaguchi, H. and Kimura, T. (1981): "Bearing capacity of footing on slopes of cohesionless soil," 16th Annual Meeting of JSSMFE, pp. 861-864 (in Japanese).

9) Shields, D. H., Scott, J. D., Bauer, G. E., Deschênes, J. H., and Barsvary, A. K. (1977): "Bearing capacity of foundations near slopes," Proc. 9th ICSMFE, Vol. 1, pp. 715-720. 
10) Sokolovski, V. V. (1960): Statics of Soil Media, Butterworths, London.

11) Terashi, M., Kitazume, M., Nakano, K. and Nakamura, T. (1986): "Effect of load eccentricity and inclination on the bearing capacity of the footing on the slope," 21st Annual Meeting of JSSMFE, pp. 1145-1148 (in Japanese).

12) Yamashita, K., Kakurai, M., Kawai, T. and Takeuchi, N. (1981): "Analysis of the ultimate bearing capacity of foundations on slopes by means of new discrete models," 16th Annual Meeting of JSS-MFE, pp. 853-856(in Japanese). 13) Yamashita, K., Saitoh, K. and Kimura, T. (1982): "Limit analysis of bearing capacity of footing on slopes of cohesionless soil," $17 \mathrm{th}$ Annual Meeting of JSSMFE, pp. 693-696 (in Japanese). 